\title{
SPIN-SELECTIVE DEPOPULATION OF TRIPLET SUBLEVELS IN RAPIDLY ROTATING TRIPLET EXCIPLEXES DETECTED BY A HEAVY-ATOM-INDUCED MAGNETIC FIELD EFFECT
}

\author{
UIIIch STEINER \\ Institut für Phy'slkalısche Chemıe der Unurersität Stuttgart, D-7000 Stuttgart 80, Germany
}

\begin{abstract}
A mechanism is presented expliunung a reported heavy-atom-induced magnetıc field efiect as a consequence of nonequilibrium triplet sublevel population in an intermediate exciplex. The triplet exciplex spin polarization is induced by sublevel-selective untersystem crossing from the exciples triplet to its single $t$ ground state and is decreased by an external magnetic field. The theory accounts almost quantitatively for the observed influence of magnetic field strength and heavyatom substituents
\end{abstract}

\section{Introduction}

Recently we reported a magnetic field effect on the radical yield of electron-transfer reactions between a dye tnplet and heavy-atom-substituted electron donors [1]. As a possible explanation we suggested that it might be due to a magnetic field modulation of gemunate radical pair recombination according to the $\Delta g$-mechanısm. Though this explanation is qualıtatively reasonable it is not supported by a quantitative application of current radical-pair theores. In a recent analysis of the $\Delta g$-mechanism, applied to the singlet recombination yield of geminate radical parrs produced in the triplet state [2], Schulten and Epstein [3] showed that w th $\Delta g=0.01$ and a magnetıc field of $10 \mathrm{kG}$, the sunglet recombination yield is of the order of $1.5 \%$, whereas we observe a magnetic field effect of about $12 \%$ at $4 \mathrm{kG}$ with a system where the $g$-factor difference is not likely to be greater than 0.01 .

In thus letter we give a prelımunary presentation of a mechanism which can quantitatıvely account for the heavy-atom-induced magneuc field effects.

\section{Kinetic model}

As previously demonstrated [4] a triplet exciplex, ${ }^{3}\left(\mathrm{AD}^{+}\right)$, of radical-parr-like structure has to be assumed as the primary product in the electron-transfer reaction between dye triplet $\left({ }^{3} \mathrm{~A}^{+}\right)$and electron donor (D):

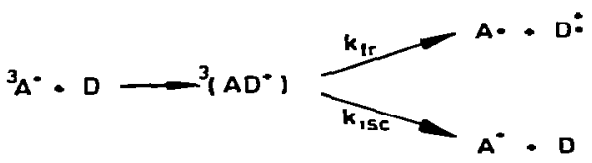

Scheme I.

Therefore, if a radical-pair mechanism fails to explain the magnetic field effect, it is tempting to invoke a magnetic field effect on the dynamics of the trplet exciplex.

Considerng the intersystem crossing process leading from the triplet to the singlet ground state, we have to regard the contributions of the triplet sublevels separately. As will be shown in a forthcoming paper [5], the three triplet substates of the exciplex are not equally effective in intersystem crossing and for simplicity we assume here that intersystem crossing occurs only from two substates (denoted $T_{x}$ and $\left.T_{y}\right)$ and is negligible from the third substate $\left(T_{z}\right)$. Of 
course the zero-field splitting of the exciplex triplet substates is rather small. Because of the dominating charge-transfer character in the cases under consideration [4], the $D$-value is expected to be of the order of $0.02 \mathrm{~cm}^{-1}$ [6], corresponding to a Zeeman splitting in a magnetuc field of about $200 \mathrm{G}$. In solutions at room temperature these levels are populated equally. However, if there is a fast intersystem crossing from two of these levels, comparable in its rate or faster than the relaxation of the triplet sublevel population, a non-equlibrium distribution over the triplet sublevels will be maintained dunng the decay of the exciplex.

The radical yield obtained under such conditions will be higher than under equilibnum conditions, since the dissociation rate constant is not expected to be spin selective. If in such a case the rate of relaxation among the triplet sublevels can be increased by some external influence, the radical yield will decrease. Sunce an external magnetic field has the effect of mixing the triplet sublevels of the randomly oriented rotating triplet exciplexes, it provides an effective additional relaxation mechanism with the consequence that the radical yield decreases with increasing magnetic field strength.

An exact treatment of the proposed mechanism should be based on the solution of the appropriate stochastic Liouville equation and will be presented elsewhere [5]. Here we treat the problem in a simple but more illustrative kinetic approximation described by:

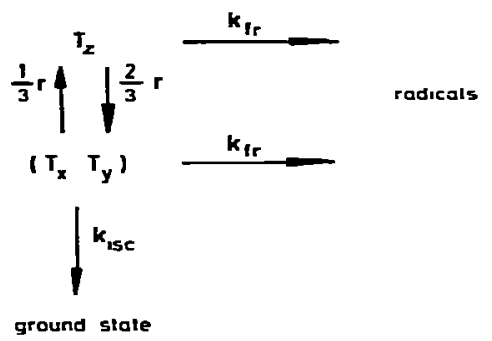

Scheme II.

It is assumed that the equilibrium of the triplet sublevel population can be described by a single rate constant $r, k_{\text {isc }}$ denotes the intersystem crossing rate constant for the substates $\mathrm{T}_{x}$ and $\mathrm{T}_{y}, k_{\mathrm{fr}}$ is the rate constant for exciplex dissociation into radicals and is assumed to be equal for $T_{x}, T_{y}$ and $T_{z}$. Since $T_{x}$ and
$T_{y}$ are not distinguished kinetically we treat them as one common state.

Assuming equal population of the triplet subleveis when the exciplex is formed, with scheme II we arrive at the following expression for the radical yield $\Phi_{\text {fr }}$,

$\Phi_{\mathrm{fr}}=k_{\mathrm{fr}} \frac{k_{\mathrm{fr}}+\frac{1}{3} k_{\mathrm{isc}}+r}{\left(k_{\mathrm{fr}}+k_{\mathrm{sc}}\right)\left(k_{\mathrm{fr}}+\frac{2}{3} r\right)+\frac{1}{3} r k_{\mathrm{fr}}}$.

As will be shown in detail elsewhere [5], the effective relaxation rate constant of the triplet sublevels in the presence of an external magnetic field is given by

$r=r_{0}\left(1+5 x^{2}+4 x^{4}\right) /\left(1+3 x^{2}+0.8 x^{4}\right)$,

where $r_{0}$ is the relaxation constant in the absence of an external field and $x$ is defined by

$x=g \mu_{\mathrm{B}} B \hbar^{-1} / r_{0}$.

Here $B$ is the magnetic field strength, $g$ the gyromagnetic ratio and $\mu_{B}$ is the Bohr magneton. In the derivation of eq. (2) the zero-field splitting of the exciplex has been neglected, so that one might expect some discrepancy between theoretical and experimental results at fields below $1 \mathbf{~ k G}$.

The relaxation constant $r_{0}$ of the triplet sublevels in zero field can be obtained by the following argument. The zero-field splitting of the exciplex corresponds to a characteristic time of approximately $\mathbf{3 0 0}$ ps which is much longer than the orientational correlation time to be expected for the exciplex in a solvent like methanol. Hence it will be a goed approximation to assume that during the rotational diffusion of the exciplex the spin remains almost fixed in the laboratory system. As a consequence the triplet sublevel relaxation time $1 / r_{0}$ should be well approxinated by the orientational relaxation time $\tau_{D}$ which can be calculated by the Debye formula [7]

$$
1 / r_{0} \approx \tau_{D}=\frac{4}{3} \pi r_{D}^{3} \eta / k T \text {, }
$$

where $r_{D}$ is the Debye radius, $\boldsymbol{\eta}$ is the solvent viscosity, $T$ the absolute temperature and $k$ Boltzmann's constant.

\section{Comparison with experiment}

To compare the predictions of our model with the experimental results we shall proceed in the following way. As expressed in eq. (1), $\Phi_{\mathrm{fr}}$ is a function of $k_{\mathrm{fr}}$, 
$k_{\mathrm{sc}}$ and $B$. We have determined $\Phi_{\mathrm{fr}}$ for a series of exciplexes with thionine as electron acceptor and various halogen anilines as electron donors [4]. As was demonstrated, halogen substitution of the anuline leaves the dissociation rate constant $k_{\mathrm{fr}}$ of the exciplex approximately unaffected but changes $k_{\text {isc }} \mathrm{sig}$ nificantly. So, if we could guess the dissociation rate constant $k_{\mathrm{fr}}$ and assume a reasonable value for $r_{\mathrm{D}}$ in eq. (4), we could calculate $k_{1 \mathrm{sc}}$ from the radical yield at zero field (henceforth denoted as $\Phi_{\mathrm{fr}}\left(k_{\mathrm{Ic}}, 0\right)$ ) by use of eqs. (1)-(4) and predict the magnetic field effect $R$, defined by

$R=100\left[\Phi_{\mathrm{fr}}\left(k_{\mathrm{isc}}, B\right)-\Phi_{\mathrm{fr}}\left(k_{\mathrm{isc}}, 0\right)\right] / \Phi_{\mathrm{fr}}\left(k_{\mathrm{isc}}, 0\right)$,

as a function of $k_{\mathrm{isc}}$ and $B$. It is indeed possible to obtain a reasonable fit of our experimental data assuming the same value of $k_{\mathrm{fr}}$ for all systems investigated. The best fit is obtained with $k_{\mathrm{fr}}=1.1 \times 10^{9} \mathrm{~s}^{-1}$ whereby the relative accuracy of the magnetic field effects detected limits the accuracy of $k_{\mathrm{fr}}$ to $\pm 30 \%$. Our calculations are based on a value of $25 \times 10^{9} \mathrm{~s}^{-1}$ for $r_{0}=$ $1 / \tau_{D}$, corresponding to a Debye radius of about $4 \AA$ in methanol.

Fig. 1 shows the magnetic field effect $R$ measured and calculated as a function of magnetic field strength for the thionine $/ p$-iodoandine triplet exciplex. Sunce we neglected the influence of the zero-field splitting, we expect a discrepancy between theory and experiment in the region of low magnetic fields. Roughly

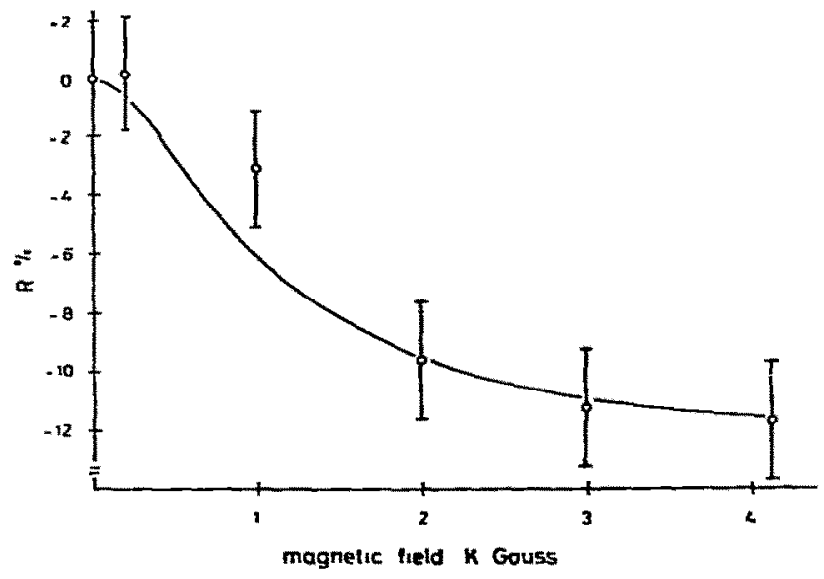

Fig. 1. Relative magnetic field effect $R$ as a function of magnetic field strength for the triplet exciplex thonune/p-iodoanune. Solud curve, calculated as described in text.

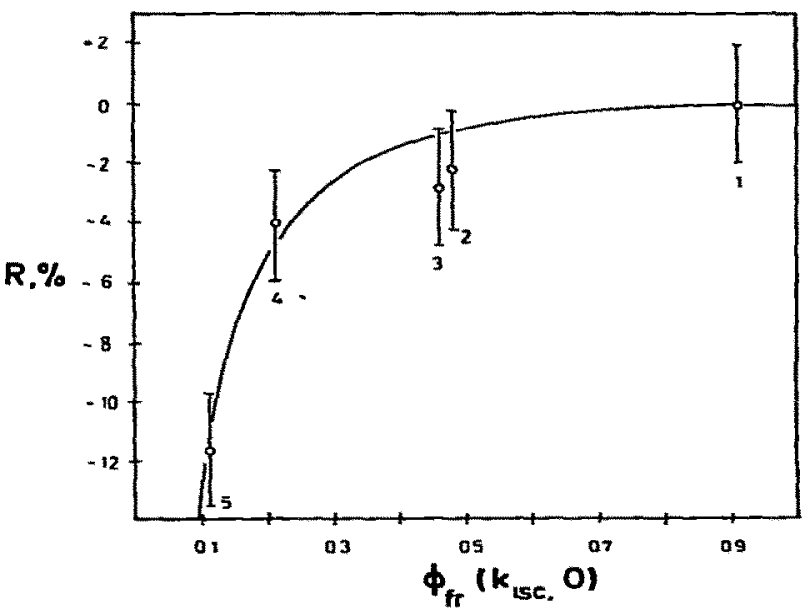

Fig. 2. Relative magnetic field effect $R$ as a function of radical yield at zero freld $\Phi_{\mathrm{T}_{\mathrm{r}}}\left(k_{\text {isc, }}, 0\right)$. The numbers refer to the duferent electron donors listed in table 1 . Solud curve, calculated as described in text.

the influence of the zero-field splitting should be that the magnetic field affects the sublevel kinetics only at values $\geqslant D$, so that, as a first-order correction, we should shift our calculated curve $\approx 200 \mathrm{G}$ to higher fields. This might explain the deviation of the point at $1 \mathrm{kG}$ where the magnetic field dependence has its steepest descent.

In fig. 2 we compare theory and expenment with respect to the influence of $k_{\mathrm{sc}}$ on the magnetic field effect. Since we obtain $k_{\mathrm{sc}}$ from $\Phi_{\mathrm{fr}}$ measured at zero field, we plot the magnetic field effect directly against $\Phi_{\mathrm{fr}}\left(k_{\mathrm{isc}}, 0\right)$ rather than against $k_{\mathrm{isc}}$. Again theory and expenment compare favorably.

In order to justify the applicability of our model, it remauns to be discussed whether the order of magnitude which had to be assumed for the exciplex dissociation rate constant is reasonable. Weller and coworkers [8] observed a dissociation rate constant of $3 \times 10^{7} \mathrm{~s}^{-1}$ for the pyrene/3,5-dimethoxy-dimethylanuline singlet exciplex in methanol. In this case the exciplex dissocrates into a pair of oppositely charged radical ions, whereas in our case only the donor radical is positively charged, so that the dissociation is not hindered by Coulomb attraction. This may explan the faster dissociation in our case where the exciplex bonding is probably only due to a resonance stablization of the charge-transfer state by the locally 
excited triplet state, $\left({ }^{3} A^{+} D\right) \leftrightarrow{ }^{3}\left(A^{+}\right)[9,10]$. Thus $k_{\mathrm{fr}} \approx 10^{9} \mathrm{~s}^{-1}$ appears physically reasonable and the good agreement between experimental and theoretical results as demonstrated in figs. 1 and 2 supports the validity of our model.

\section{Concluding remarks}

It may be anticipated that spin-orbit-couplingenhanced magnetic field effects of the kind reported here will become a valuable tool to study the propertes and dynamics of short-lived triplet exciplexes. Since the orientational relaxation time provides an absolute time basis for all rate processes shown in scheme II, we can now obtain absolute rate constants for the intersystem crossing of the triplet exciplexes. The values obtained for the systems described in this paper are listed in table 1 , their relative accuracy, as in the case of $k_{\mathrm{fr}}$, amounting to $\pm 30 \%$.

Refining the theoretical treatment and improving the experimental accuracy should allow determination of the zero-field splitting parameter of these and similar systems which cannot be studied by ESR experiments. Thus detaled information on the electronic structure of short-lived intermediates in electrontransfer reactions with excited triplet states may become avaulable.

It should be noted that scheme II provides a general mechanism for magnetic field effects; it is not necessanly restricted to triplet exciplexes but may involve any triplet-state species provided it decays by a fast sublevel-selective intersystem crossing process com-

Table 1

Radical yields $\Phi_{\mathrm{fr}}$ and untersystem crossung rate constants $k_{15 c}$ for triplet exciplexes between thionine and various electron donors

\begin{tabular}{llc}
\hline Donor a) & $\Phi_{\mathrm{fr}_{\mathrm{r}}} \mathrm{b}$ & $\left.k_{\mathrm{ISc}} \mathrm{c}\right)$ \\
\hline aniline (1) & 0.91 & 0.15 \\
$p$-Br-aniline (2) & 0.48 & 1.8 \\
$m$-I-anuline (3) & 0.46 & 2.0 \\
$o$-I-aniline (4) & 0.21 & 6.8 \\
$p$-I-anilune (5) & 0.11 & 16.3 \\
\hline
\end{tabular}

a) Numbers in parentheses refer to data points in fig. 2.

b) Measured at zero field.

c) $10^{9} \mathrm{~s}^{-1}$, accuracy $\pm 30 \%$, calculation based on $k_{\mathrm{fr}_{\mathrm{r}}}=1.1 \mathrm{X}$ $10^{9} \mathrm{~s}^{-1}$. peting with a non-sublevel-selective second decay mechanism. Whereas in our case the second decay channel is provided by the exciplex dissociation into radicals, processes like hydrogen-atom or protontransfer may function as the second channel in other cases.

Finally we point out that the model presented here bears a close relation to a mechanism of electron spin polarization in photochemically generated radicals as suggested by Atkins and Evans [11]. In their mechanism the different population rate constants of the spin sublevels of a reacting triplet are chiefly important. Significant effects ensuc if the quenching reaction of the triplet, leading to radical formation, is faster than the relaxation of the triplet Zeeman level population $\left(T_{1}\right)$. In our case, however, the sublevel population of the precursor triplets $\left({ }^{3} A^{+}\right)$has already obtained thermal equilibrium when the exciplexes are formed (in order to avoid reaction of the excited dye singlets we generally choose fairly iow donor concentrations so that the effective lifetime of ${ }^{3} A^{+}$is $\approx 100$ $1000 \mathrm{~ns})$. The non-equilibrium sublevel population of the exciplex triplet, ${ }^{3}\left(\mathbf{A D}^{+}\right)$, is produced by its own spin-selective depopulation process. It may be concluded, however, that in our case, too, the radicals formed should show spin polarization, and it might be of interest to look for CIDEP effects in these systems.

\section{Acknowledgement}

The author is indebted to Professor Albert Weller for drawing his attention to the possible role of the exciplex triplet sublevels in the magnetic field effect. Thanks are due to Professor H.E.A. Kramer for his interest and support of this work. The advice of $\mathrm{Dr}$. D. Miller in translating the manuscript is gratefully acknowledged.

\section{References}

[1] U. Steiner, Z. Naturforsch. 34a (1979) 1093.

[2] H. Staerk and K. Razi Naqvi, Chem. Phys. Letters 50 (1977) 386.

[3] K. Schulten and I.R. Epstein, J. Chem. Phys. 71 (1979) 309.

[4] U. Steiner and G. Winter, Chem. Phys. Letters 55 (1978) 364. 
[5] U. Stemer, to be published.

[6] H. Beens, J. de Jong and A. Weller, Colloque Ampère 15, ed. P. Averbuch (North-Holland, Amsterdam, 1969) pp. 289-292;

H. Hayashi, S. Iwata and S. Nagakura, J. Chem. Phys. 50 (1969) 993.

[7] P. Debye, Polare Molekeln (Leupzig, 1929).

[8] K. Schulten, H. Staerk, A, Weller, H,-J, Wernes and B. Nickel, Z. Physık. Chem. NF 101 (1976) 371,
H.-J. Wemer, H. Staerk and A. Weller, J. Chem. Phys. 68 (1978) 2419.

[9] A. Weller, in: The exciplex, eds. M. Gordon and W.R. Ware (Academc Press, New York, 1975) pp. 23-30.

[10] U. Steiner, Schweratome als molekulare Sonden zum Nachweıs und Studium des Verhaltens von Tnplettevciplexen (Hochschulverlag, Freiburg, 1979).

[11] P.W. Atkins and G.T. Evans, Mol. Phys. 27 (1974) 1633. 